\title{
Nonlinear temporal pulse cleaning techniques and application
}

Yi Xu, Jianzhou Wang, Yansui Huang, Yanyan Li, Xiaomin Lu, and Yuxin Leng

State Key Laboratory of High Field Laser Physics, Shanghai Institute of Optics and Fine Mechanics, Chinese Academy of Sciences, Shanghai 201800, China

(Received 27 June 2012; revised 3 September 2012; accepted 8 October 2012)

\begin{abstract}
Two different pulse cleaning techniques for ultra-high contrast laser systems are comparably analysed in this work. The first pulse cleaning technique is based on noncollinear femtosecond optical-parametric amplification (NOPA) and second-harmonic generation (SHG) processes. The other is based on cross-polarized wave (XPW) generation. With a double chirped pulse amplifier (double-CPA) scheme, although temporal contrast enhancement in a high-intensity femtosecond Ti:sapphire chirped pulse amplification (CPA) laser system can be achieved based on both of the techniques, the two different pulse cleaning techniques still have their own advantages and are suitable for different contrast enhancement requirements of different laser systems.
\end{abstract}

Keywords: pulse cleaning, temporal contrast, chirped pulse amplification

\section{Introduction}

The development of high-intensity femtosecond Ti:sapphire chirped pulse amplification (CPA) lasers has made it possible to realize ultra-intense and ultra-fast lasers with a focused intensity exceeding $10^{20} \mathrm{~W} / \mathrm{cm}^{2}$ in small-scale laboratorie ${ }^{[1-4]}$. Based on ultra-intense and ultra-fast lasers, many exciting opportunities for studying laser-matter interactions in the relativistic dominated regime have been created $^{[5-7]}$. For laser-matter interaction experiments at such an intense level, the temporal contrast should be at least $10^{10}$ to restrict destructive pre-plasma dynamics ${ }^{[8]}$. However, in a typical high-intensity Ti:sapphire CPA laser, the temporal contrast is usually only $7-8$ orders of magnitude due to the amplified spontaneous emission noise or the unwanted pre-pulses. This contrast level is insufficient to support high-intensity physics experiments in the relativistic dominated regime. Therefore, enhancement of temporal contrast has become a very crucial problem for developing high-intensity laser physics in recent years.

Contrast improvement in Ti:sapphire CPA laser systems can be achieved through pulse cleaning technique development. One solution is to place a pulse cleaner after the final compressor stage, such as a plasma mirror ${ }^{[9]}$ and harmonic frequency generation ${ }^{[10]}$. Such a pulse cleaner can suppress all noise structures simultaneously, including the amplified

Correspondence to: Yuxin Leng, State Key Laboratory of High Field Laser Physics, Shanghai Institute of Optics and Fine Mechanics, Chinese Academy of Sciences, Shanghai 201800, China. Email: lengyuxin@mail. siom.ac.cn spontaneous emission (ASE) noise and leaked pre-pulses. However, it also introduces obvious energy loss. Unfortunately, such energy loss cannot be compensated generally since the pulse cleaner is the last element in the laser chain. The other solution is to use a double-CPA setup including a pulse cleaner between the two CPA systems. The advantage of this scheme is that seed pulses generated by the pulse cleaner have sufficient energy and contrast to support contrast enhancement in the laser system. Further, the energy loss introduced by the pulse cleaner can be recovered by the subsequent CPA stages. Recently, several pulse cleaning techniques, based on the double-CPA scheme, have been reported and applied to develop highcontrast high-intensity femtosecond Ti:sapphire CPA lasers, such as saturable absorber ${ }^{[11,12]}$, XPW generation ${ }^{[13-15]}$ and $\mathrm{NOPA}^{[16,17]}$.

In this work, with a double-CPA scheme, a pulse cleaning technique based on NOPA and SHG processes is applied to improve the contrast in our TW-level Ti:sapphire CPA laser system. Further, temporal contrast enhancement with a pulse cleaner based on XPW generation is also experimentally investigated. Based on both of the pulse cleaners, the temporal contrast of the TW-level Ti:sapphire CPA laser can be promoted efficiently. Finally, the application prospects of the two different pulse cleaning techniques in ultra-high contrast laser systems are comparably analysed. 


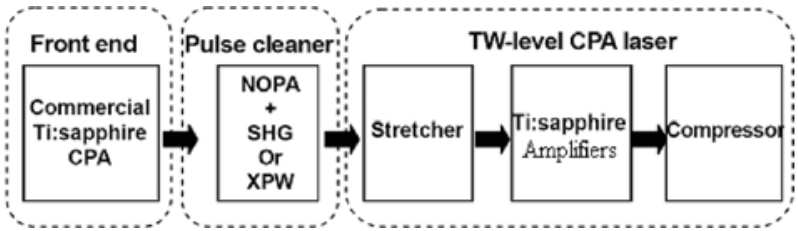

Figure 1. Simplified layout of a high-intensity femtosecond Ti:sapphire laser system with a double-CPA scheme.

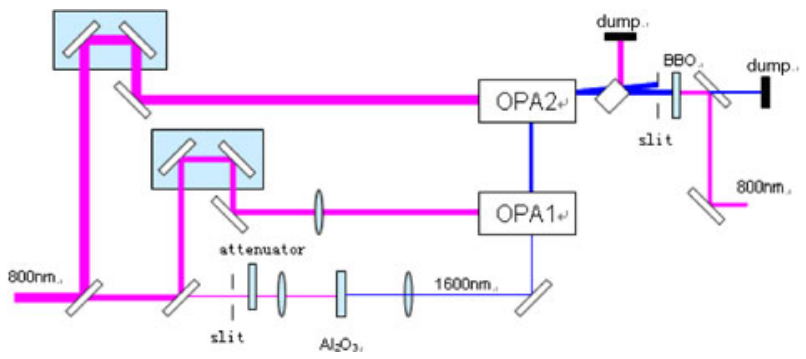

Figure 2. Setup of pulse cleaner based on NOPA and SHG processes.

\section{High-contrast Ti:sapphire CPA laser with pulse cleaner based on NOPA and SHG processes}

A simplified layout of a high-intensity femtosecond Ti: sapphire laser system with a double-CPA scheme is shown in Figure 1. The system consists of two successive Ti: sapphire CPA stages linked by a pulse cleaner.

A pulse cleaner based on NOPA and SHG processes has been reported and applied to a TW-level Ti:sapphire CPA laser in our previous work ${ }^{[18,19]}$. In this work, we modify this technique and apply it for contrast improvement again. The scheme of the modified pulse cleaner is described in Figure 2. The commercial Ti:sapphire CPA laser (Spectra Physics, Spitfire-35) can deliver $800 \mathrm{~nm}$, sub-40 fs pulses with $3.8 \mathrm{~mJ}$ energy at $1 \mathrm{kHz}$ repetition rate for the pulse cleaner. In contrast to our previous pulse cleaner, this pulse cleaner consists of four parts: (1) a white-light continuum (WLC) generation stage, generating broadband seed pulses; (2) a WLC-seeded IR OPA stage, generating broadband signal or idler pulses near $1.6 \mu \mathrm{m}$; (3) an IR OPA stage seeded by the signal pulses from the first stage OPA generating infrared signal pulses with energy of $600 \mu \mathrm{J}$; (4) an SHG crystal, generating cleaned femtosecond pulses by frequency doubling the infrared pulses. After the SHG process $(0.1 \mathrm{~mm}$ thick BBO plate), $120 \mu \mathrm{J}$ cleaned pulses are generated in $800 \mathrm{~nm}$ working wavelength. The pulse duration of the cleaned pulse is about 33 fs. Thanks to these nonlinear processes (especially the SHG process), significant enhancement of temporal contrast can be achieved for this pulse cleaner. The temporal contrast of the cleaned pulse is better than $10^{11}$ (measurement limited), as shown (dark curve) in Figure 3.

The second CPA system, operating at $10 \mathrm{~Hz}$, consists of a stretcher, a regenerative amplifier, two stages of multi-pass amplifiers, and a pulse compressor in a vacuum chamber. After being stretched, the seed pulses are amplified to $0.75 \mathrm{~J}$ before entering the compressor. The laser pulse duration is then compressed to 55 fs (FWHM) by the compressor. The compressed pulse energy is about $380 \mathrm{~mJ}$, corresponding to a peak power of about $7 \mathrm{TW}^{[19]}$.

The temporal contrast of the high-contrast TW-level Ti:sapphire CPA laser and that of our original TW-level Ti:sapphire CPA laser are measured with a scanning thirdorder cross-correlator (Amplitude Technologies, Sequia). Figure 4 presents the comparable contrast curves. By employing a double-CPA scheme including this novel pulse cleaner, the temporal contrast of the TW-level Ti:sapphire CPA laser can be promoted from the original $\sim 10^{8}$ to $\sim 10^{11}$ within the time window of -480 ps to -100 ps. And as further amplification has very small influence on the temporal contrast of the output pulse ${ }^{[14]}$, we can believe that this pulse cleaning technique can also support a highcontrast Ti:sapphire CPA laser system at higher peak power.

\section{High-contrast Ti:sapphire CPA laser with pulse cleaner based on XPW generation}

XPW generation is a four-wave mixing process: the XPWgenerated wave has the same wavelength as the input pulse and a cubic dependence on the intensity. Meanwhile, the polarization of the XPW-generated wave is crossed with the input pulse. Consequently, it can be discriminated from the input pulse or the low-intensity noise via two cross polarizers, and therefore can be used to improve the temporal contrast of the input pulse. In this work, a pulse cleaner based on XPW generation is also designed and applied to improve the temporal contrast of our Ti:sapphire CPA laser ${ }^{[15]}$. The scheme of the pulse cleaner is shown in Figure 5.

The XPW setup is composed of a neutral attenuator, two crossed Glan laser polarizers, one focusing lens and one collimating focus mirror (both with a $1.7 \mathrm{~mm}$ focal length), and two $\mathrm{BaF}_{2}$ crystals (crystallographic orientation [011], $2 \mathrm{~mm}$ thickness). After passing through the attenuator, the energy of the injected initial pulse decreases to $450 \mu \mathrm{J}$. The focus of the lens is placed into a small vacuum chamber to avoid nonlinear effects in air. Taking into account the stability, after optimizing the positions and the rotation of the nonlinear crystals, and the chirp of the initial pulse, a cleaned seed pulse with energy of $60 \mu \mathrm{J}$ is obtained, corresponding to a conversion efficiency of $13.3 \%$. The cleaned seed pulse is injected into the TW-level Ti:sapphire CPA laser, and an output pulse with temporal contrast of $\sim 10^{11}$ is achieved (as shown in Figure 6). The experimental result also demonstrates the contrast enhancement of the Ti:sapphire CPA laser. 


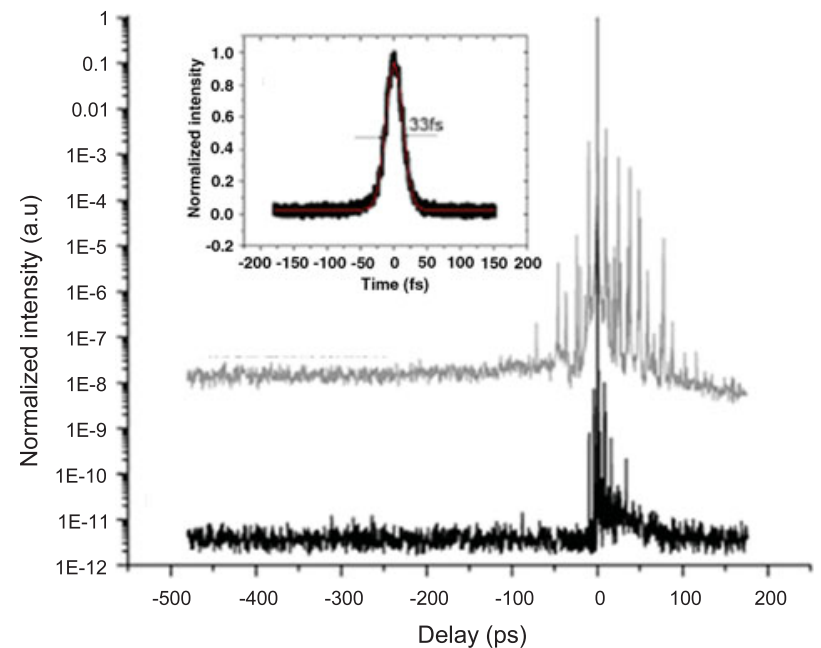

Figure 3. Contrast curves of the commercial CPA laser (grey curve) and the pulse cleaner based on NOPA and SHG (dark curve). The inset is the pulse duration of the cleaned pulse.

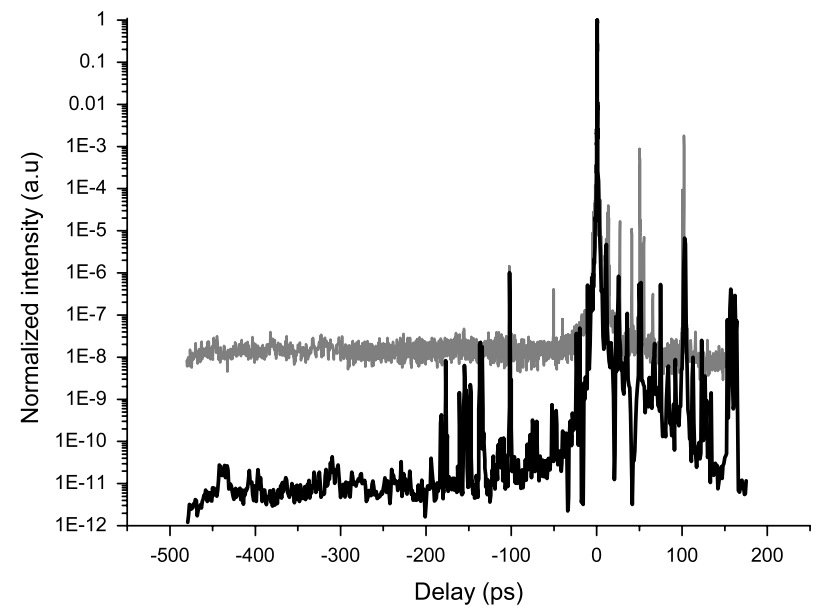

Figure 4. Contrast curves of the high-contrast TW-level Ti:sapphire CPA laser (black curve) and the original TW-level Ti:sapphire CPA laser (grey curve). The pre-pulses are identified to be the artefacts generated by multiple reflections at the optical components in the cross-correlator and amplifiers.

\section{Application prospects of the two different pulse cleaning techniques in an ultra-high contrast laser system}

XPW generation has become a well-known pulse cleaning technique; it has been widely investigated and applied to improve temporal pulse contrast in recent years. However, a pulse cleaner based on NOPA and SHG processes is a novel method, and this is the first time it has been applied to a TWlevel Ti:sapphire CPA laser. Our high-contrast Ti:sapphire laser system with a pulse cleaner based on NOPA and SHG processes has shown good performance during daily operation. Recently, based on this high-contrast TW-level Ti:sapphire CPA laser, a proton generation experiment with nanometre film targets has been carried out. The experimental results also demonstrate the enhancement of the contrast.

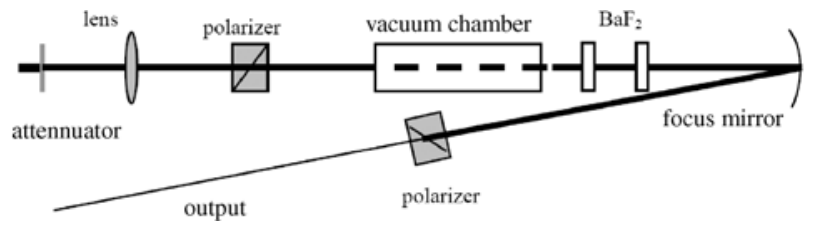

Figure 5. Setup of pulse cleaner based on XPW generation ${ }^{[15]}$.

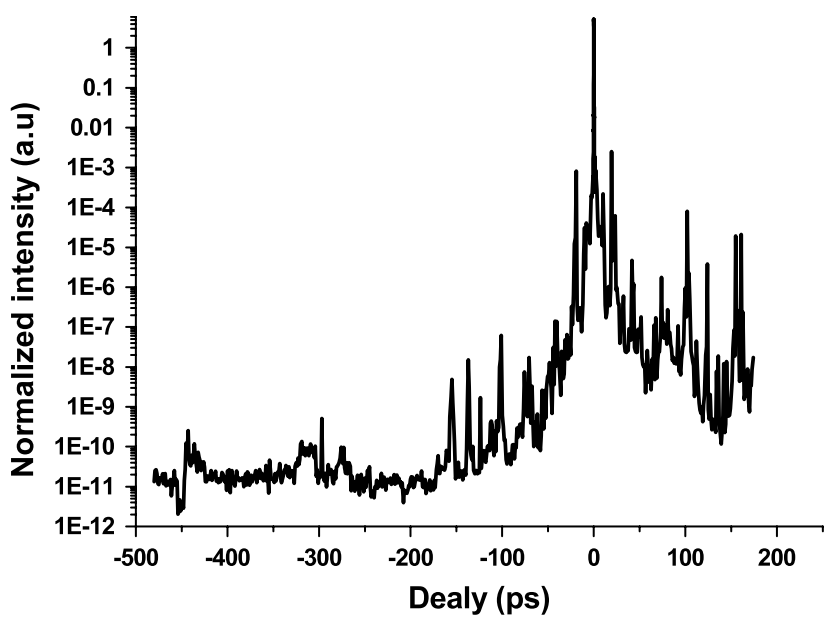

Figure 6. Contrast curves of the high-contrast Ti:sapphire CPA laser with a pulse cleaner based on XPW generation ${ }^{[15]}$.

The purpose of this work is to improve the temporal contrast in Ti:sapphire CPA lasers and to search for a pulse cleaning technique which can support ultra-high contrast lasers for ultra-high focused intensity in the near future. As the temporal contrast of a Ti:sapphire CPA laser is largely determined by the contrast and energy of the seed pulse ${ }^{[19,20]}$, we will discuss the application prospects of the two different pulse cleaning techniques according to the cleaned pulse energy and contrast.

First, XPW generation is a third-order nonlinear process; the temporal contrast of the XPW-generated wave should reach a very high level in theory. Assume that the contrast of the initial pulse is $C_{0}$; then the contrast of the XPWgenerated wave should be $C_{1}=\eta^{*} C_{0}^{3}$, where $\eta$ is the conversion efficiency of the XPW generation. However, the contrast of a pulse cleaner based on XPW generation is actually limited by the extinction of the two crossed polarizers, and contrast enhancement is usually limited at 4-5 orders of magnitude. NOPA and SHG are secondorder nonlinear processes; the contrast of a pulse cleaner based on NOPA and SHG processes can be estimated as $C_{1}=\eta^{*}\left(g^{*} C_{0}\right)^{2}$ in theory, where $g$ is the gain of the NOPA process and $\eta$ is the conversion efficiency of the SHG process. The contrast enhancement of this pulse cleaner is not limited by any optical element. Thus, the contrast enhancement is at least 7 orders of magnitude for a initial pulse contrast of $10^{7}$. Therefore, the temporal contrast of a pulse cleaner based on NOPA and SHG processes can be 
expected to reach a higher level than that of a pulse cleaner based on XPW generation.

Second, to achieve a third-order nonlinear process, $\mathrm{BaF}_{2}$ crystals should be placed near the focus to reach the adequate peak intensity for XPW generation. To avoid damage, XPW generation works with only a limited input energy, of the order of $1 \mathrm{~mJ}$ order ${ }^{[21]}$. Until now, it has only been demonstrated for microjoule-level output pulse even for conversion efficiency exceeding $20 \%$. As well as the limited temporal contrast, the limited output pulse energy of XPW generation is also a problem when developing an ultrahigh contrast laser system, which is because the contrast of the Ti:sapphire CPA laser commonly increases linearly with the seed pulse energy ${ }^{[19]}$. Though NOPA and SHG processes have a low conversion efficiency (about 10\%), they have no limitation to the input pulse energy. Thanks to the quick development of high-energy, high-repetition femtosecond pulse amplification technology, commercial $10 \mathrm{~mJ}, 1 \mathrm{kHz}$ Ti:sapphire CPA laser systems have become available, and recently $20 \mathrm{~mJ}, 1 \mathrm{kHz}$ Ti:sapphire CPA lasers are also being quickly developed. A pulse cleaner based on NOPA and SHG processes can generate a cleaned pulse with higher energy (even to millijoule level) if a higherenergy CPA front-end is used. Millijoule-level cleaned seed pulses can be directly amplified in the Ti:sapphire power amplifier after being stretched. Then gain narrowing and contrast deterioration in high-gain amplifiers can be avoided. Of course, with such a high-energy front-end, the cost of the whole laser system will increase correspondingly.

Compared to a pulse cleaner based on NOPA and SHG processes, a pulse cleaner based on XPW generation also has its own advantages: simple setup, low cost, and broadened spectrum via a third-order nonlinear process. Therefore, it may be a good choice for developing Ti:sapphire CPA lasers with temporal contrast of $10^{11}-10^{12}$. However, due to the limited contrast enhancement and output energy, if the contrast of the first CPA stage is not sufficiently high, it will be difficult for a pulse cleaner based on XPW generation to support contrast better than $10^{12}$ in a double-CPA scheme. The pulse cleaner based on NOPA and SHG processes has the potential advantage of producing a seed pulse with higher energy and higher contrast. It can be applied to develop an ultra-high contrast laser system for ultra-high focused intensity in the future.

\section{Conclusion}

Two different nonlinear temporal pulse cleaning techniques (NOPA with SHG processes and XPW generation) are applied to improve the temporal contrast of a high-intensity femtosecond Ti:sapphire laser system with a double-CPA scheme. Although contrast enhancement can be achieved based on both of the two pulse cleaning techniques, there still exists an optimized choice for different contrast enhancement requirements of different laser systems. A pulse cleaner based on XPW generation is sufficient to support the development of a high-intensity femtosecond Ti:sapphire CPA laser with temporal contrast of $10^{11-12}$ generally. But if higher contrast is required, a pulse cleaner based on NOPA and SHG processes may have more advantages. Further, a combination of different pulse cleaning techniques is also regarded as a solution for the higher contrast requirements of higher intensity femtosecond laser systems.

\section{References}

1. X. Y. Liang, Y. X. Leng, C. Wang, C. Li, L. H. Lin, B. Z. Zhao, Y. H. Jiang, X. M. Lu, M. Y. Hu, C. M. Zhang, H. H. Lu, D. J. Yin, Y. L. Jiang, X. Q. Lu, H. Wei, J. Q. Zhu, R. X. Li, and Z. Z. Xu, Opt. Express 15, 15335 (2007).

2. M. Aoyama, K. Yamakawa, Y. Akahane, J. Ma, N. Inoue, H. Ueda, and H. Kiriyama, Opt. Lett. 28, 1594 (2003).

3. V. Yanovsky, V. Chvykov, G. Kalinchenko, P. Rousseau, T. Planchon, T. Matsuoka, A. Maksimchuk, J. Nees, G. Cheriaux, G. Mourou, and K. Krushelnick, Opt. Express 16, 2109 (2008).

4. S. W. Bahk, P. Rousseau, T. Planchon, V. Chvykov, G. Kalintchenko, A. Maksimchuk, G. Mourou, and V. Yanovsky, Opt. Lett. 29, 2837 (2004).

5. S. V. Bulanov, T. Esirkepov, D. Habs, F. Pegoraro, and T. Tajima, Eur. Phys. J. D 55, 483 (2009).

6. G. A. Mourou, T. Tajima, and S. V. Bulanov, Rev. Mod. Phys. 78, 309 (2006).

7. L. L. Ji, B. F. Shen, D. X. Li, D. Wang, Y. X. Leng, X. M. Zhang, M. Wen, W. P. Wang, J. C. Xu, and Y. H. Yu, Phys. Rev. Lett. 105, 025001 (2010).

8. D. Umstadter, Phys. Plasmas 8, 1774 (2001).

9. C. Thaury, F. Quéré, J. P. Geindre, A. Levy, T. Ceccotti, P. Monot, M. Bougeard, F. Réau, P. d'Oliveira, P. Audebert, R. Marjoribanks, and P. Martin, Nat. Phys. 3, 424 (2007).

10. S. Mironov, V. Lozhkarev, V. Ginzburg, I. Yakovlev, G. Luchinin, E. Khazanov, and A. Sergeev, Proc. SPIE 7721, 77211R (2010).

11. H. Kiriyama, M. Mori, Y. Nakai, T. Shimomura, M. Tanoue, A. Akutsu, H. Okada, T. Motomura, S. Kondo, S. Kanazawa, A. Sagisaka, J. L. Ma, I. Daito, H. Kotaki, H. Daido, S. Bulanov, T. Kimura, and T. Tajima, Opt. Commun. 282, 625 (2009)

12. H. Kiriyama, M. Mori, Y. Nakai, T. Shimomura, H. Sasao, M. Tanoue, S. Kanazawa, D. Wakai, F. Sasao, H. Okada, I. Daito, M. Suzuki, S. Kondo, K. Kondo, A. Sugiyama, P. R. Bolton, A. Yokoyama, H. Daido, S. Kawanishi, T. Kimura, and T. Tajima, Opt. Lett. 35, 1497 (2010).

13. A. Jullien, O. Albert, F. Burgy, G. Hamoniaux, J. P. Rousseau, J. P. Chambaret, F. A. Rochereau, G. Chériaux, J. Etchepare, N. Minkovski, and S. M. Saltie, Opt. Lett. 30, 920 (2005).

14. V. Chvykov, P. Rousseau, S. Reed, G. Kalinchenko, and V. Yanovsky, Opt. Lett. 31, 1456 (2006).

15. J. Z. Wang, Y. S. Huang, Y. Xu, Y. Y. Li, X. M. Lu, and Y. X. Leng, Acta Phys. Sin. 61, 094214 (2012).

16. C. Dorrer, I. A. Begishev, A. V. Okishev, and J. D. Zuegel, Opt. Lett. 32, 2143 (2007).

17. C. Shah, R. P. Johnson, T. Shimada, K. A. Flippo, J. C. Fernandez, and B. M. Hegelich, Opt. Lett. 34, 2273 (2009).

18. Y. S. Huang, C. M. Zhang, Y. Xu, D. X. Li, Y. X. Leng, R. X. Li, and Z. Z. Xu, Opt. Lett. 36, 1 (2011).

19. Y. Xu, Y. S. Huang, Y. Y. Li, J. Z. Wang, X. M. Lu, Y. X. Leng, R. X. Li, and Z. Z. Xu, IEEE J. Quantum Electron. 48, 516 (2012).

20. V. Bagnoud, J. D. Zuegel, N. Forget, and C. Le Blanc, Opt. Express 15, 5504 (2007).

21. L'. Veisz, in Coherent and Ultrashort Pulse Laser Emission, F. J. Duarte, ed. (Intech, 2010), p. 305, Chap. 14. 